\title{
Brillouin light scattering study of microscopic structure and dynamics in pyrrolidinium based ionic liquids
}

Supti Das, ${ }^{{ }^{*} \dagger+}$ Dhanya Radhakrishnan, ${ }^{2}$ Venkata. S. Bhadram, ${ }^{3}$ Chandrabhas Narayana ${ }^{2}$ and Aninda J. Bhattacharyya ${ }^{1 *}$

${ }^{1}$ Solid State and Structural Chemistry Unit, Indian Institute of Science, Bangalore 560012, India

${ }^{2}$ Chemistry and Physics of Materials Unit, Jawaharlal Nehru Centre for Advanced Scientific Research, Jakkur P.O., Bangalore 560064, India

${ }^{3}$ IMPMC, Sorbonne Université, CNRS, MNHN, 4, place Jussieu, 75005 Paris, France

Keywords: Brillouin Light scattering, Room Temperature Ionic Liquids, N-alkyl-N-methyl pyrrolidinium bis(trifluoromethanesulfonyl) imide $\left(\mathrm{P}_{1 n} \mathrm{TFSI}\right)$, Organic Ionic plastic crystalline materials, Mechanical property

\begin{abstract}
Pyrrolidinium based ionic liquids are known to be good ionic conductors even in solid-state around room temperature, which is attributed to the highly disordered plastic crystalline phase. Moreover, these ionic liquids are characterized by multiple phase transitions which include plastic, structural glass, and glassy crystal phases with varying levels of molecular disorder. Temperature-dependent Brillouin light scattering is used to investigate the phase transitions in a series of alkylmethylpyrrolidinium
\end{abstract}


Bis(trifluoromethanesulfonyl) imides ( $\mathrm{P}_{1 n}$ TFSI, $\left.\mathrm{n}=1,2,4\right)$. Brillouin spectral features such as the number of acoustic modes, their shape, and linewidth provide the picture of different disordered phases resultant of dynamics at the microscopic scale. The longitudinal and transverse acoustic velocities in different phases are determined from the corresponding acoustic mode frequencies (Brillouin shift). Extremely low acoustic velocities in the solid phase of $\mathrm{P}_{11}$ TFSI and $\mathrm{P}_{12}$ TFSI are a consequence of a high degree of disorder and plasticity present in the system. Anomalous temperature-dependent behavior of linewidth and asymmetric (Fano) line shape of acoustic modes observed in certain phases of $\mathrm{P}_{1 n}$ TFSI could be due to the strong coupling between the Brillouin central peak and the acoustic phonons. The present results establish that the Brillouin light scattering technique can be efficiently used to understand the complex phase behavior, microscopic structure, and dynamics of ionic liquids.

\section{Introduction}

Ionic Liquids (ILs) are the generic term for a class of molten organic salts which are fluids at temperatures around $100{ }^{\circ} \mathrm{C}$ or below. ${ }^{1}$ They normally comprise of an organic cation and an inorganic anion. The class of low melting point ILs which is liquid at ambient/room temperature is called room temperature molten salts or room temperature ionic liquids (RTILs). RTILs have stimulated much interest in a variety of chemical, physical and biological processes in the past few decades due to their remarkable properties such as negligible volatility, thermal \& electrochemical stability, nonflammability, varying viscosity, and high ionic conductivity. Another interesting aspect of these ILs is that their physico-chemical properties could be tuned by varying the combinations of cations, anions, and alkyl chain length or the substituents on the cation. On the other hand, there is a class of materials, which are solid-state analogues of ILs, known as organic ionic plastic crystals (OIPCs) have gained much interest over well-known organic molecular plastic crystals due to their negligible vapour pressure even in the molten state. ${ }^{1 \mathrm{c}-\mathrm{e}, 2}$ Usually, in their solid-state, they show plastic crystalline behavior attributed to the rotational motion or orientational disorder (short-range disorder) with long-range translational order (i.e. individual molecules maintain their lattice positions). OIPCs display significant 
structural disorder over a wide temperature range. They are characterized by the presence of one or more solid-solid (S-S) transitions below melting which is attributed to the onset of rotational or orientational motions, indicating the transformation from low-temperature ordered crystalline phase to high-temperature disorder structures. Therefore, the combined advantages of both plastic crystals as well as ILs have triggered the applications of OIPCs as green and safe electrolytes in a diverse range of electrochemical energy technologies including high energy density rechargeable batteries (e.g. all-solidstate batteries, metal-ion, metal sulfur, or metal- $\left.\mathrm{O}_{2}\right),{ }^{3}$ supercapacitors, ${ }^{4}$ fuel cells ${ }^{5}$, and solar cells. ${ }^{6}$

The worldwide demand for clean energy has intensified the development of novel electrolytes for next-generation energy devices. Therefore, research on developing new OIPCs as well as RTILs has gained considerable attention during the past decade. One of the promising series of ILs is based on Nalkyl N-methylpyrrolidinium cation $\left(\mathrm{P}_{1 n}, \mathrm{n}=1-4\right)$ and the bis(trifluoromethanesulfonyl) imide (TFSI) anion. $^{1 \text { c-e, } 7}$ The combination of $\mathrm{P}_{l n}$ cation with a more diffuse charge anion (TFSI) produces a hydrophobic ionic liquid series with a relatively high ionic conductivity even in solid-state along with a wide electrochemical stability window. These series of ILs $\left(\mathrm{P}_{1 n} \mathrm{TFSI}\right)$ at room temperature could form both OIPCs $\left(e . g . \mathrm{P}_{1 n}\right.$ TFSI for $\left.\mathrm{n}=1,2\right)$ as well as RTILs $\left(e . g ., \mathrm{P}_{1 n} \text { TFSI, } \mathrm{n}=3,4 \ldots\right)^{1 \mathrm{c}}$ depending upon the length of alkyl chain substituent on the cation. Pyrrolidinium based ILs have been well investigated by several research groups in next-generation energy storage devices such as Li-Air battery ${ }^{3 \mathrm{~b}, 3 \mathrm{~d}, \mathrm{e}}$ where other ILs failed in terms of stability. Previous study based on differential electrochemical mass spectrometry reveals that electrochemical stabilities and discharge capacities are comparable between pyrrolidinium and 1,2-dimethoxyethane (DME), and pyrrolinidium based ILs performs even better than DME in Li-O battery. $^{8}$ To efficiently utilize these ILs in various applications, it is very important to understand their physical properties with respect to varying external factors such as temperature, pressure, etc. These ILs undergo multiple S-S phase transitions with respect to temperature and detailed knowledge of these phase transitions, corresponding (structural) disorder and different relaxation processes are essential to understand the physico-chemical properties of these ILs and to enlarge their 
applicability in various fields. Therefore, lot of focus has been given lately to interpret the relation between the transport properties and various relaxation processes in ILs. ${ }^{1 c, 3 a, 9}$ In particular, the dynamics and relaxations of RTILs have gained much attention and have been studied using dielectric relaxation spectroscopy, ${ }^{9 \mathrm{a}}$ Kerr effect spectroscopy, ${ }^{10}$ infrared, ${ }^{11}$ Raman ${ }^{12}$ NMR relaxation, quasielastic neutron scattering, ${ }^{13}$ terahertz spectroscopy, ${ }^{14}$ etc. However, fewer studies used Brillouin scattering to probe the microscopic dynamics or relaxations across various phase transitions in RTILs. ${ }^{15}$

Brillouin light scattering (BLS) is a non-contact technique used to probe the acoustic and relaxation modes in the gigahertz $(\mathrm{GHz})$ range. Analysis of Brillouin spectral features gives information on the mechanical properties, acoustic attenuation, relaxation processes, etc. Being a non-destructive technique, BLS could be used to study a variety of materials ranging from bulk solids or liquids to composites and thin films. In imidazolium RTILs, the domain formation on increasing the alkyl chain length was demonstrated using temperature-dependent BLS along with dielectric loss spectroscopy. ${ }^{15}$ BLS was also used to study the plastic crystal-liquid transition in archetypal organic molecular plastic crystal, succinonitrile. ${ }^{16}$ The influence of solvent dynamics on the ion transport mechanism across ordered crystal to disordered plastic crystal phase transition in pure succinonitrile and Li-salt: succinonitrile plastic crystalline electrolytes was also illustrated using BLS. ${ }^{17}$

Here, we investigate a series of ionic liquids composed of alkylmethylpyrrolidinium bis(trifluoromethanesulfonyl) imide ( $\mathrm{P}_{1 n}$ TFSI, $\mathrm{n}=1,2$ and 4; Figure $\mathrm{S} 1$ ) using temperature dependent BLS. These ILs display a variety of phases which includes glassy, supercooled liquid, crystalline, plastic crystalline, etc. over a wide range of temperature. Using BLS, the influence of the alkyl chain length on the disorder, plasticity, and ion transport properties has been investigated. The phase transitions in these materials were also identified by studying the changes in the acoustic modes (frequency, shape \& number) and their linewidths (full with at half maximum, FWHM). We attempt to demonstrate BLS as a useful tool to establish the correlation between lattice disorder and ion transport in ILs, and to differentiate between glassy, plastic crystalline, and crystalline phases. 


\section{Experimental materials and methods}

N,N-dimethylpyrrolidinium bis(trifluoromethanesulfonyl) imide $\quad\left(\mathrm{P}_{11}\right.$ TFSI) and N-Methyl-Nethylpyrrolidinium bis(trifluoromethanesulfonyl) imide $\left(\mathrm{P}_{12}\right.$ TFSI) were synthesized by metathesis reaction from the corresponding iodide salts according to the work reported in literature ${ }^{1 \mathrm{c}}$ and the NMR characterization is presented in supporting information (Figure S1). N-methyl-N-butylpyrrolidinium bis(trifluorosulfonyl) imide ( $\mathrm{P}_{14}$ TFSI, purity $98.5 \%$ Sigma Aldrich (fluka)) was used as received from the suppliers. Differential scanning calorimetry (DSC) has been used to measure the thermal transitions of the ILs. Samples for DSC measurements were loaded (under argon) in hermetically sealed aluminum crucibles, and data was collected (Mettler Toledo DSC $823^{\mathrm{e}}$ ) with a heating rate of $5{ }^{\circ} \mathrm{C} / \mathrm{min}$. Brillouin spectra were recorded in the back-scattering geometry using $532 \mathrm{~nm}$ laser excitation from a single-mode diode pumped Nd-YAG laser (Coherent, USA, Model No. DPSS 532-400) with a power of $\sim 10 \mathrm{~mW}$, focused on a spot size of $\sim 20 \mu \mathrm{m}$. The scattered light was analyzed using a 3+3 pass tandem FabryPerot interferometer (JRS Scientific Inc., 116 Switzerland), which has a finesse of $\sim 100$ and is equipped with a photo avalanche diode (Perkin Elmer, Canada) as the detector. A free spectral range (FSR) of $25 \mathrm{GHz}$ was used to measure the longitudinal (LA) and transverse (TA) acoustic modes in $\mathrm{P}_{14}$ TFSI while both $25 \mathrm{GHz}$ (for LA) and $12 \mathrm{GHz}$ (for TA) were used for measuring these modes in $\mathrm{P}_{11}$ TFSI and $\mathrm{P}_{12}$ TFSI. Samples were inserted in a capillary tube and both ends were sealed before fixing it to the heating stage using suitable heat-transmitting grease. Temperature-dependent measurements are obtained using a liquid nitrogen-cooled cryostage (Linkam stage, THMS 600) and the temperature was measured with an accuracy of $\pm 0.1 \mathrm{~K}$. The samples were cooled from the room temperature, and Brillouin experiments were performed on heating. Typical spectral accumulation time ranges from 20 to 2 minutes as the temperature is increased. The peak frequency and linewidth (FWHM) of the Brillouin modes were extracted by non-linear least-square fitting of the data with a Lorentzian or Fano resonance function, ${ }^{18}$ along with the central peak.

\section{Results and Discussions:}




\section{Phase transitions from the thermal analysis:}

The pyrrolidinium based ILs undergoes multiple S-S phase transitions including crystalline, plastic, and glassy phases before finally melting. Typical DSC data $\mathrm{P}_{1 n}$ TFSI, $\mathrm{n}=1,2$, and 4 ionic liquids are shown in Figure 1 and it agrees well with the previous reports. ${ }^{1 \mathrm{c}, 19}$ The standard convention of nomenclature has been used to number the phases, such as, phase-I is the highest temperature solid phase prior to melting and is followed by lower temperature phases which are numbered as phase-II, phase-III etc. ${ }^{19 \mathrm{~b},}$ 20 DSC thermogram of $\mathrm{P}_{11}$ TFSI exhibits two very weak endothermic peaks, within the studied temperature range, which is attributed to $\mathrm{S}-\mathrm{S}$ transitions and the sample melts at $\mathrm{T}_{\mathrm{I}_{\rightarrow}} \sim 135{ }^{\circ} \mathrm{C}$. These solid phases are represented as phase-III, phase-II and phase-I with transition temperatures at $\mathrm{T}_{\mathrm{III} \rightarrow \mathrm{II}^{\mathrm{II}}} \sim 20$ ${ }^{\circ} \mathrm{C}$ and $\mathrm{T}_{\mathrm{II}_{\rightarrow}} \mathrm{I} 77^{\circ} \mathrm{C}$, respectively. $\mathrm{P}_{12} \mathrm{TFSI}$ also shows several solid phases (phase-V to phase-I) prior to

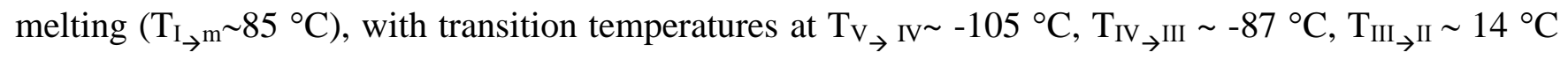
and, $\mathrm{T}_{\mathrm{II}_{\rightarrow} \mathrm{I}} \sim 44{ }^{\circ} \mathrm{C}$. Compared to other ILs, $\mathrm{P}_{14}$ TFSI exhibits a glassy phase (phase-IV) which upon heating transforms into a supercooled liquid (phase-III) at $\mathrm{T}_{\mathrm{g}} \sim-86^{\circ} \mathrm{C}$. The exotherm at $-55^{\circ} \mathrm{C}$ is related to the crystallization of the supercooled liquid into a metastable phase and subsequent transitions occur at $\sim-45^{\circ} \mathrm{C}\left(\mathrm{T}_{\text {meta }_{\rightarrow} \text { II }}\right)$ and $\sim-20{ }^{\circ} \mathrm{C}\left(\mathrm{T}_{\mathrm{II}_{\rightarrow}} \mathrm{I}\right)$ before melting at $-9{ }^{\circ} \mathrm{C}$. At present, the nature of phase-II and phase-I in $\mathrm{P}_{14}$ TFSI could not be established.

\section{Brillouin spectra analysis:}

Figure 2 shows Brillouin spectra of $\mathrm{P}_{1 n} \operatorname{TFSI}(\mathrm{n}=1,2$, and 4$)$ at room temperature $\left(25^{\circ} \mathrm{C}\right)$ as well as at temperatures where they are in a molten state (liquid) and solid-state (phase-I). At room temperature,

the spectrum consists of a single longitudinal acoustic (LA) mode (Figure 2(A)). While $\mathrm{P}_{12}$ TFSI and $\mathrm{P}_{11}$ TFSI are plastic crystals at $25{ }^{\circ} \mathrm{C}, \mathrm{P}_{14}$ TFSI with the longer alkyl chain is in the liquid phase. Consequently, LA mode softens (decrease in frequency) on increasing the alkyl chain length $(n=1 \rightarrow 4)$. Due to the existence of different phases in these ionic compounds, a better understanding of their properties can be obtained by comparing them in the same physical states. Figure 2(B) shows the 
Brillouin spectra of these ILs in their liquid state. The spectra display a well resolved LA mode which hardens (increase in frequency) from $5.5 \mathrm{GHz}$ to $8.5 \mathrm{GHz}$ as the alkyl chain length is increased. The linewidth also increases from $1.5 \mathrm{GHz}$ to $1.9 \mathrm{GHz}$ as the chain length is increased from $\mathrm{n}=1$ to 4 , but a comparable linewidth is observed for $\mathrm{P}_{14}$ TFSI $(1.9 \mathrm{GHz})$ and $\mathrm{P}_{12}$ TFSI $(1.86 \mathrm{GHz})$. Figure 2 (C) corresponds to their solid phase (phase-I) and the frequency of LA modes shows the order, $\mathrm{P}_{12}$ TFSI (8.8 $\mathrm{GHz})<\mathrm{P}_{11}$ TFSI $(9 \mathrm{GHz})<\mathrm{P}_{14}$ TFSI $(13.6 \mathrm{GHz})$. Additionally, the linewidth of these ILs follows the order, $\mathrm{P}_{12}$ TFSI $(1.75 \mathrm{GHz})>\mathrm{P}_{11}$ TFSI $(1.55 \mathrm{GHz})>\mathrm{P}_{14}$ TFSI $(1.11 \mathrm{GHz})$.

Brillouin shift or acoustic mode frequency $\left(v_{B}\right)$ can be used to determine the acoustic velocity (V) using the expression $v_{B}=2 V \mu / \lambda$ in the backscattering geometry, where $\mu$ and $\lambda$ are the refractive index and wavelength of the incident light. Studies on few pyrrolidinium ionic liquids show that the refractive index of ionic liquids has a negligible influence on alkyl chain length. ${ }^{21}$ We have considered the refractive index of 1.423 for the liquid phase $\mathrm{e}^{22}$ and 1.8 for the solid phases ${ }^{23}$ of all ILs. As determined, the acoustic velocity in liquid phase increases from $1060 \mathrm{~m} / \mathrm{s}$ to $1650 \mathrm{~m} / \mathrm{s}$, upon increasing the alkyl chain length (Table 1). This implies that the bulkier cation entity results in a less compressible and rigid system. In phase-I, the LA velocity of $\mathrm{P}_{11}$ TFSI and $\mathrm{P}_{12}$ TFSI are nearly equal ( $\left.1360 \mathrm{~m} / \mathrm{s}\right)$, but are much lower when compared to $\mathrm{P}_{14} \mathrm{TFSI}(\sim 2040 \mathrm{~m} / \mathrm{s})$. In addition to the LA modes shown in Figure 2, one or more TA modes denoted as $\mathrm{TA}_{1}$ (slow) and $\mathrm{TA}_{2}$ (fast) are also observed in the solid phases when the spectrum is measured using a lower free spectral range (Figure 3, Figure S2). In phase-I, $\mathrm{P}_{11} \mathrm{TFSI}$ exhibits two TA modes at $3.0\left(\mathrm{TA}_{1}\right)$ and $3.8\left(\mathrm{TA}_{2}\right)$, and these modes occur at 5.9 and $7.2 \mathrm{GHz}$, respectively in $\mathrm{P}_{14}$ TFSI, while only one TA mode $(2.9 \mathrm{GHz})$ is observed in $\mathrm{P}_{12}$ TFSI. The velocity of slowest TA mode is $\sim 440 \mathrm{~m} / \mathrm{s}$ in $\mathrm{P}_{11}$ TFSI and $\mathrm{P}_{12}$ TFSI, and is significantly lower than that of $\mathrm{P}_{14}$ TFSI $(865 \mathrm{~m} / \mathrm{s})$. In fact, the LA and TA velocities of $\mathrm{P}_{11}$ TFSI and $\mathrm{P}_{12}$ TFSI in phase-I is much lower when compared to succinonitrile (LA and TA velocities are $\sim 2200 \mathrm{~m} / \mathrm{s}$ and $\sim 850$, respectively) ${ }^{16}$ as well as other molecular solids. ${ }^{24}$ The rotational disorder of individual ions in the plastic phase results in liquidlike behavior including diffusion which could lead to low mechanical strength. The poor mechanical 
strength is often associated with the plasticity as observed in similar OIPCs such as N-methyl-Npropyl pyrrolidinium hexafluorophosphate. ${ }^{25}$ The low acoustic velocity in $\mathrm{P}_{11}$ TFSI and $\mathrm{P}_{12}$ TFSI, thus indicates high disorder which could originate from the rotational motion of one or two ions of the ILs. Moreover the low transverse acoustic (TA) velocities, here, implies that these ILs possess extremely low shear modulus and hence impart low resistance to shear stress. This observation corroborates with the high plasticity of $\mathrm{P}_{11}$ TFSI and $\mathrm{P}_{12}$ TFSI at room temperature.

Furthermore, the linewidth of the Brillouin mode $(\Gamma)$ can be used to estimate the viscosity, $\left.\eta \approx \rho \Gamma / 2 q^{2}\right)$ or attenuation coefficient, $\alpha=\Gamma / 2 V$. Here $\rho, q(=2 \mu / \lambda)$ are the mass density and scattering wave vector, respectively. The viscosity of $\mathrm{P}_{14}$ TFSI determined from the linewidth is 90 $\mathrm{mPa} / \mathrm{s}$, (obtained using $\rho=1394 \mathrm{~kg} / \mathrm{m}^{3}$ ) which is in good agreement with the previous studies. ${ }^{1 \mathrm{c},}{ }^{26}$ But we could not estimate the viscosity of $\mathrm{P}_{11}$ TFSI and $\mathrm{P}_{12}$ TFSI due to unavailability of their density values in the molten state. In the solid phase, a correlation can be made between linewidth and the disorder which is attributed to the large size and concentration of vacancies as well as the rotational degree of freedom of molecules. ${ }^{27}$ This disorder also gives rise to the high ionic conductivity exhibited by these ILs in one or more of their solid phases. ${ }^{1 c, 28}$ The $\mathrm{P}_{1 n}$ TFSI series of ionic liquids studied here are known to have high ionic conductivity in one or more of their solid phases. ${ }^{1 c}$ In phase-I, $\mathrm{P}_{12}$ TFSI exhibits the highest conductivity as well as the highest disorder compared to $\mathrm{P}_{11}$ TFSI and $\mathrm{P}_{14}$ TFSI. Moreover, at any given temperature the conductivity of these ILs decreases with the decrease in alkyl chain length ${ }^{27 b}$ and this agrees well with the observed trend in the linewidth at room temperature discussed above.

\section{Temperature-dependent Brillouin spectra:}

Brillouin spectra of ILs obtained at various temperatures, comprising the several phase transitions, spanning from melting to glass/crystalline are shown in Figure 3. The acoustic modes show significant changes in both peak positions and linewidth around these phase transitions. The LA mode in solid and liquid phases is represented by LA-S ( $\mathrm{S}$ denote solid-state), and LA-L (L denote liquid state), 
respectively in Figure 3B, D, F. The S-L phase transitions in all the samples are characterized by an abrupt decrease in the frequency shift of LA mode as well as the disappearance of the TA modes in the liquid phase.

In $\mathrm{P}_{11}$ TFSI, LA and TA mode frequencies increase gradually with decreasing temperature and exhibit a change in the slope near $\mathrm{T}_{\mathrm{III}-\mathrm{II}}$ between crystalline and plastic phases, while only negligible changes are observed near $\mathrm{T}_{\mathrm{II}-\mathrm{I}}$ between plastic crystalline phases. The hardening of the acoustic modes upon decreasing the temperature indicates the increase in the associated elastic/mechanical properties. $\mathrm{P}_{12}$ TFSI also displays clear changes in the Brillouin shift of the acoustic modes during the phase transitions, similar to $\mathrm{P}_{11}$ TFSI. Upon heating, a clear change in slope could be observed near $\mathrm{T}_{\mathrm{V}-\mathrm{IV}}$ which will be explained later. No major changes are observed in the acoustic mode frequencies during the transitions between plastic crystalline phases (phases-III, II, and I) in $\mathrm{P}_{12}$ TFSI except for the transition from phase-III to phase-II near $25{ }^{\circ} \mathrm{C}$. The LA mode of $\mathrm{P}_{12}$ TFSI near $25{ }^{\circ} \mathrm{C}\left(\sim \mathrm{T}_{\text {III-II, }}\right.$ Figure 3(C)) could be deconvoluted into two modes (LA-S1=9.5 and LA-S2=10.4 GHz), indicating the coexistence of two plastic phases. This suggests that $\mathrm{P}_{12}$ TFSI is more disordered and highly conducting plastic crystal than $\mathrm{P}_{11}$ TFSI at room temperature. This observation agrees well with the literature where the positron annihilation lifetime spectroscopy (PALS) ${ }^{27 a}$ experiment reveals more rotational freedom and vacancy size in $\mathrm{P}_{12}$ TFSI as compared to $\mathrm{P}_{11}$ TFSI. Similar to LA modes, TA modes also harden on lowering the temperature but while two TA modes are observed in phase-III and IV, only one could be recognized in phase-II and phase-I. Additionally, both $\mathrm{P}_{12}$ TFSI and $\mathrm{P}_{11}$ TFSI show sluggishness during melting as evidenced by the coexistence of LA-S and LA-L after melting for over a temperature range of $20^{\circ} \mathrm{C}$ and $10^{\circ} \mathrm{C}$, respectively.

The Brillouin shift of LA and TA modes of $\mathrm{P}_{14}$ TFSI increases only slightly as the temperature is decreased from phase-I to III, indicating its rigid structure even in the supercooled liquid state (phaseIII). But an abrupt softening of the LA is observed in the glassy phase below $\mathrm{T}_{\mathrm{g}}$ (phase-IV). This is contradictory to the observed temperature dependence across the $\mathrm{T}_{\mathrm{V}-\mathrm{IV}}$ in $\mathrm{P}_{12} \mathrm{TFSI}$, which is also 
depicted as $\mathrm{T}_{\mathrm{g}}$ in some reports. ${ }^{1 \mathrm{c}} \mathrm{P}_{12}$ TFSI is a room temperature plastic crystal phase and on cooling, the rotational disorder is quenched in phase $\mathrm{V}$, resulting in a 'glassy crystal'. ${ }^{1 c,}{ }^{29}$ On further cooling, this glassy state relaxes and achieves orientational and positional ordering ${ }^{29}$ as seen in the XRD studies. ${ }^{19 a}$, ${ }^{29}$ Whereas in $\mathrm{P}_{14}$ TFSI, cooling it from the room temperature liquid phase results in the formation of 'structural glass' which is both orientationally and translationally disordered. Heating $\mathrm{P}_{14}$ TFSI above $\mathrm{T}_{\mathrm{g}}$ results in a supercooled liquid phase whereas $\mathrm{P}_{12}$ TFSI transforms into a plastic crystal in phase-III. The higher degree of disorder present in structural glass results in the lower LA shifts compared to the glassy crystal (phase-V) of $\mathrm{P}_{12}$ TFSI and other plastic phases. This shows that temperature-dependent BLS could be used as a tool to efficiently distinguish the different phases in particular the glassy phases. Interestingly, TA modes in $\mathrm{P}_{14}$ TFSI is observed only in phase-I and II indicating the existence of highly disordered supercooled liquid and glassy phases at low temperatures similar to the glassy crystal phase in $\mathrm{P}_{12}$ TFSI.

In order to understand the microscopic dynamics of these IL systems, it is required to correlate the Brillouin data with the structural origin of different rotar phases. By comparing Figures 3 with thermal data (Figure 1), it is observed that high plasticity (i.e, low acoustic velocity) in the solid-state is a phenomenon strongly present in $\mathrm{P}_{1 n}$ TFSI with smaller alkyl chains (methyl \& ethyl). Therefore it appears that the behavior is more likely to be associated with the cation. Generally, molecules would require a considerable free volume in their surrounding area in order to rotate around any of the obvious axes. Pyrrolidinium ring is close to planar (see Scheme S1) and has several axes of rotation which might be active in the solid plastic phases. Particularly, rotation about an axis normal to the ring is possible with an accessible energy barrier for the smaller alkyl substituents. On the other hand for larger alkyl substituents (butyl) the alkyl side chain may become too bulky to allow free rotation about such an axis and the rotator phases are thus not easily accessible. Hence, $\mathrm{P}_{11}$ TFSI and $\mathrm{P}_{12}$ TFSI display higher degree of rotation and higher plasticity than $\mathrm{P}_{14}$ TFSI. 
Furthermore, the dynamics of molecular disorder of these ionic liquids can be observed from the temperature variation of the linewidth of LA modes (Figure 4). For all the samples, a significant change in the magnitude of linewidth is observed in the proximity of phase transitions. In $\mathrm{P}_{11}$ TFSI, the linewidth increases on decreasing temperature in phase-I and II. Similar to mode frequencies, linewidth

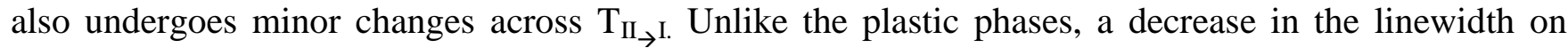
reducing temperature is observed in the crystalline phase-III which results in a peak-like feature at $\mathrm{T}_{\text {III }_{\rightarrow} \mathrm{II}}$ as shown in Figure $4 \mathrm{~A}$. Similar to $\mathrm{P}_{11}$ TFSI, linewidth increases on decreasing the temperature in the plastic phases (phase-III, II and I) of $\mathrm{P}_{12}$ TFSI, and displays changes across these transitions. Compared to $\mathrm{P}_{11}$ TFSI, these changes are significant and indicate the higher degree of disorder in the plastic phases of $\mathrm{P}_{12}$ TFSI which is in concurrence with the faster dynamics exhibited by this system. However, the decrease in linewidth on decreasing the temperature below phase-IV indicates the anharmonic phonon relaxations similar to the ordered phase (phase-III) in $\mathrm{P}_{11}$ TFSI. On the other hand, an abrupt increase in the linewidth across $\mathrm{T}_{\mathrm{g}}$ is observed in $\mathrm{P}_{14}$ TFSI (Figure 4C). This indicates an increase in the disorder resulting from an increase in the positional as well as rotational degrees of freedoms as it enters a supercooled liquid state from the structural glassy phase. Thereafter the linewidth decreases with an increase in temperature, similar to $\mathrm{P}_{11}$ TFSI and $\mathrm{P}_{12}$ TFSI, and undergoes only minor changes across phase transitions.

Comparing the temperature dependence of linewidth it is evident that the increase in the linewidth on decreasing the temperature is a characteristic behaviour in the plastic crystal phase. This is contrary to the usual anharmonic relaxation behaviour of acoustic phonons where linewidth of phonon modes decrease upon cooling that is normally observed in the liquid, ordered crystal, disordered glassy crystal, and structural glass phases. This anomalous increase in the linewidth could also originate from the coupling between the acoustic phonons and the central peak $(\mathrm{CP})$. The $\mathrm{CP}$ in the Brillouin spectra appears as a peak which is centered at zero frequency and its width could be used to determine various phonon relaxation times. Generally, many materials exhibit one or more $\mathrm{CP}$ corresponding to different 
relaxation processes such as thermal, rotational, structural relaxation, etc. ${ }^{30}$ The $\mathrm{CP}$ along with the LA modes in the liquid phases of these ILs is shown in Figure 5A. The linewidth of the CP increases from 2 $\mathrm{GHz}$ to $10 \mathrm{GHz}$ with an increase in the alkyl chain length from $\mathrm{P}_{11}$ to $\mathrm{P}_{14}$. The linewidth of the $\mathrm{CP}$ is similar in phase-I and liquid phase in all the systems (Figure S3) indicating that its origin could be related to the hopping or diffusion of the ions, which is in agreement with the XRD studies. ${ }^{30-31}$ But the temperature evolution of $\mathrm{CP}$ could not be tracked in other low-temperature phases due to its weak intensity or because of the low spectral range used. Nevertheless, the presence of $\mathrm{CP}$ at low temperature is evident from the asymmetric lineshape of LA modes originating due to the coupling between CP and acoustic mode (Figure 5B). The asymmetric lineshape could also contribute towards the anomalous damping of the LA modes observed at low temperatures. The asymmetric line shape of the LA mode can be quantified by using Fano lineshape in the peak-fitting process. The Fano function along the central peak is given by the expression

$$
y=y_{0}+A\left[\left(q+\left(\frac{2\left(\omega-\omega_{C}\right)}{\Gamma_{1}}\right)\right)^{2} /\left(1+\left(\frac{2\left(\omega-\omega_{C}\right)}{\Gamma_{1}}\right)^{2}\right)\right]+B \frac{\Gamma_{2}}{4 \omega^{2}+\Gamma_{2}^{2}}
$$

Where $\omega_{\mathrm{c}}, \omega, \Gamma_{1}, \Gamma_{2}$, q, A and B are the resonance frequency, frequency, FWHM of the LA, FWHM of the $\mathrm{CP}$, asymmetry parameter, and constants, respectively. The low value of $\mathrm{q}$ suggests a higher asymmetry and indicates a higher phonon-CP coupling whereas a higher q indicates a lower coupling and hence the lineshape is close to Lorentzian. Due to the narrowness of CP in $\mathrm{P}_{11} \mathrm{TFSI}$, the LA modes can be fitted with a Lorentzian function throughout the whole temperature range. However, Fano line shape was used for the data in the temperature range $\sim 100$ to $10{ }^{\circ} \mathrm{C}$ in $\mathrm{P}_{12} \mathrm{TFSI}$ (i.e., phase-IV to phaseIII) and -90 to $-30{ }^{\circ} \mathrm{C}$ (i.e., phase-III to phase-II) in $\mathrm{P}_{14}$ TFSI. All the other phases in $\mathrm{P}_{12}$ TFSI and $\mathrm{P}_{14}$ TFSI could be fitted with Lorentzian function., The value of $\mathrm{q}$ increases from 3 to 9 in $\mathrm{P}_{12}$ TFSI (phase-IV to phase-III) and from $\sim 2$ to 8 in $\mathrm{P}_{14}$ TFSI (phase-III to phase-II) with an increase in temperature. Relatively low values of Fano parameter suggests the presence of strong CP-phonon 
coupling at low temperature. In general, IL freezes to a more compact conformation below the melting temperature where ions are localized via pinning to the lattice. This pinning effect increases the probability of CP-phonon coupling. ${ }^{17}$ Furthermore, the steric hindrance attributed to the increase of the size of the side chain induces more coupling in $\mathrm{P}_{14}$ TFSI. Hence, the immobile and bulkier $\mathrm{P}_{14}{ }^{+}$ion contributes to a higher CP-phonon coupling and asymmetric line shape at low temperature in the case of $\mathrm{P}_{14}$ TFSI. The presence of strong CP-phonon coupling in the plastic phases of $\mathrm{P}_{12}$ TFSI and $\mathrm{P}_{14}$ TFSI could explain the anomalous behavior of linewidth in these phases shown in Figure 4.

\section{Conclusions}

Brillouin light scattering has been used to study the structural and disorder dynamics of various lowtemperature phases of ionic liquids $\mathrm{P}_{11}$ TFSI, $\mathrm{P}_{12}$ TFSI, and $\mathrm{P}_{14}$ TFSI. Ranging from glassy, crystalline, and plastic crystalline, these materials undergo several phase transitions before melting. The temperature dependent changes in the Brillouin shift and linewidth was successfully employed to differentiate these phases. Our study envisages that $\mathrm{P}_{12}$ TFSI is highly dynamic and disordered compared to others which results in its high plasticity and ionic conductivity. The present study enriches the understanding of complex phase behavior and ion transport dynamics in ILs. We anticipate that it would widen the scope of Brillouin light scattering technique in providing insights into the fundamental relaxation processes in ionic salts which would facilitate their applicability in various technologies.

ASSOCIATED CONTENT

Supporting Information. 


\section{Corresponding Authors}

*Professor Aninda J Bhattacharyya; email: anindajb@iisc.ac.in; Tel: +91 80 22932616; Fax: +91 80 23601310

*Dr. Supti Das; email: dassupti@gmail.com, ORCID iD:_https://orcid.org/0000-0002-7107-0968

\section{Present Addresses}

${ }^{\dagger}$ Department of Chemistry, University of Oslo, P.O. Box 1033 Blindern, NO-0315 Oslo, Norway

\section{Author Contributions}

The manuscript was written through contributions of all authors. All authors have given approval to the final version of the manuscript.

${ }^{\ddagger}$ These authors contributed equally.

ACKNOWLEDGMENT

S. Das thanks CSIR and D. R. thanks UGC for Research Fellowships. 


\section{REFERENCES}

1. (a) Galiński, M.; Lewandowski, A.; Stępniak, I., Ionic liquids as electrolytes. Electrochim. Acta 2006, 51 (26), 5567-5580; (b) Fukaya, Y.; Ohno, H., Hydrophobic and polar ionic liquids. PCCP 2013, 15 (11), 4066-4072; (c) MacFarlane, D.; Meakin, P.; Sun, J.; Amini, N.; Forsyth, M., Pyrrolidinium imides: a new family of molten salts and conductive plastic crystal phases. J. Phys. Chem. B 1999, 103 (20), 4164-4170; (d) Forsyth, C.; MacFarlane, D.; Golding, J.; Huang, J.; Sun, J.; Forsyth, M., Structural characterization of novel ionic materials incorporating the bis (trifluoromethanesulfonyl) amide anion. Chem. Mater. 2002, 14 (5), 2103-2108; (e) Golding, J.; Hamid, N.; MacFarlane, D.; Forsyth, M.; Forsyth, C.; Collins, C.; Huang, J., N-methyl-N-alkylpyrrolidinium hexafluorophosphate salts: novel molten salts and plastic crystal phases. Chem. Mater. 2001, 13 (2), 558-564.

2. (a) Pringle, J. M.; Howlett, P. C.; MacFarlane, D. R.; Forsyth, M., Organic ionic plastic crystals: recent advances. J. Mater. Chem. 2010, 20 (11), 2056-2062; (b) Pringle, J. M., Recent progress in the development and use of organic ionic plastic crystal electrolytes. PCCP 2013, 15 (5), 1339-1351.

3. (a) Lewandowski, A.; Świderska-Mocek, A., Ionic liquids as electrolytes for Li-ion batteriesan overview of electrochemical studies. J. Power Sources 2009, 194 (2), 601-609; (b) Grande, L.; Paillard, E.; Kim, G.-T.; Monaco, S.; Passerini, S., Ionic Liquid Electrolytes for Li-Air Batteries: Lithium Metal Cycling. Int. J. Mol. Sci. 2014, 15 (5), 8122-8137; (c) Kuboki, T.; Okuyama, T.; Ohsaki, T.; Takami, N., Lithium-air batteries using hydrophobic room temperature ionic liquid electrolyte. $J$. Power Sources 2005, 146 (1), 766-769; (d) Kar, M.; Simons, T. J.; Forsyth, M.; MacFarlane, D. R., Ionic liquid electrolytes as a platform for rechargeable metal-air batteries: a perspective. $P C C P$ 2014, 16 (35), 18658-18674; (e) Elia, G. A.; Hassoun, J.; Kwak, W.-J.; Sun, Y.-K.; Scrosati, B.; Mueller, F.; Bresser, D.; Passerini, S.; Oberhumer, P.; Tsiouvaras, N., An Advanced Lithium-Air Battery Exploiting an Ionic Liquid-Based Electrolyte. Nano Lett. 2014, 14 (11), 6572-6577; (f) Jin, L.; Howlett, P. C.; Pringle, J. M.; Janikowski, J.; Armand, M.; MacFarlane, D. R.; Forsyth, M., An organic ionic plastic crystal electrolyte for rate capability and stability of ambient temperature lithium batteries. Energy Environ. Sci. 2014, 7 (10), 3352-3361. 
4. Taniki, R.; Matsumoto, K.; Nohira, T.; Hagiwara, R., All solid-state electrochemical capacitors using N,N-dimethylpyrrolidinium fluorohydrogenate as ionic plastic crystal electrolyte. J. Power Sources 2014, 245, 758-763.

5. (a) Rana, U. A.; Vijayaraghavan, R.; MacFarlane, D. R.; Forsyth, M., An organic ionic plastic crystal electrolyte based on the triflate anion exhibiting high proton transport. Chem. Commun. 2011, 47 (22), 6401-6403; (b) Luo, J.; Conrad, O.; Vankelecom, I. F. J., Imidazolium methanesulfonate as a high temperature proton conductor. J. Mater. Chem. A 2013, 1 (6), 2238-2247.

6. (a) Cao-Cen, H.; Zhao, J.; Qiu, L.; Xu, D.; Li, Q.; Chen, X.; Yan, F., High performance all-solidstate dye-sensitized solar cells based on cyanobiphenyl-functionalized imidazolium-type ionic crystals. J. Mater. Chem. 2012, 22 (25), 12842-12850; (b) Armel, V.; Forsyth, M.; MacFarlane, D. R.; Pringle, J. M., Organic ionic plastic crystal electrolytes; a new class of electrolyte for high efficiency solid state dye-sensitized solar cells. Energy Environ. Sci. 2011, 4 (6), 2234-2239.

7. (a) Jin, L.; Howlett, P.; Efthimiadis, J.; Kar, M.; Macfarlane, D.; Forsyth, M., Lithium doped N, N-dimethyl pyrrolidinium tetrafluoroborate organic ionic plastic crystal electrolytes for solid state lithium batteries. J. Mater. Chem. 2011, 21 (27), 10171-10178; (b) Adebahr, J.; Johansson, P.; Jacobsson, P.; MacFarlane, D.; Forsyth, M., Ab initio calculations, Raman and NMR investigation of the plastic crystal di-methyl pyrrolidinium iodide. Electrochim. Acta 2003, 48 (14), 2283-2289; (c) Borodin, O.; Smith, G. D., Structure and dynamics of N-methyl-N-propylpyrrolidinium bis (trifluoromethanesulfonyl) imide ionic liquid from molecular dynamics simulations. J. Phys. Chem. B 2006, 110 (23), 11481-11490.

8. Das, S.; Højberg, J.; Knudsen, K. B.; Younesi, R.; Johansson, P.; Norby, P.; Vegge, T., Instability of Ionic Liquid-Based Electrolytes in Li-O2 Batteries. J. Phys. Chem. C 2015, 119 (32), 18084-18090.

9. (a) Hunger, J.; Stoppa, A.; Schrödle, S.; Hefter, G.; Buchner, R., Temperature dependence of the dielectric properties and dynamics of ionic liquids. ChemPhysChem 2009, 10 (4), 723-733; (b) 
MacFarlane, D. R.; Pringle, J. M.; Howlett, P. C.; Forsyth, M., Ionic liquids and reactions at the electrochemical interface. PCCP 2010, 12 (8), 1659-1669.

10. Shirota, H.; Funston, A. M.; Wishart, J. F.; Castner Jr, E. W., Ultrafast dynamics of pyrrolidinium cation ionic liquids. J. Chem. Phys. 2005, 122 (18), 184512.

11. Shi, F.; Deng, Y., Abnormal FT-IR and FTRaman spectra of ionic liquids confined in nanoporous silica gel. Spectrochim. Acta A Mol. Biomol. Spectrosc. 2005, 62 (1), 239-244.

12. (a) Ribeiro, M., Low-frequency Raman spectra and fragility of imidazolium ionic liquids. $J$. Chem. Phys. 2010, 133 (2), 024503-024503; (b) Faria, L. F.; Matos, J. R.; Ribeiro, M. C., Thermal Analysis and Raman Spectra of Different Phases of the Ionic Liquid Butyltrimethylammonium Bis (trifluoromethylsulfonyl) imide. J. Phys. Chem. B 2012, 116 (30), 9238-9245.

13. Triolo, A.; Russina, O.; Hardacre, C.; Nieuwenhuyzen, M.; Gonzalez, M. A.; Grimm, H., Relaxation processes in room temperature ionic liquids: The case of 1-butyl-3-methyl imidazolium hexafluorophosphate. J. Phys. Chem. B 2005, 109 (46), 22061-22066.

14. Asaki, M.; Redondo, A.; Zawodzinski, T.; Taylor, A., Dielectric relaxation and underlying dynamics of acetonitrile and 1-ethyl-3-methylimidazolium triflate mixtures using $\mathrm{THz}$ transmission spectroscopy. J. Chem. Phys. 2002, 116 (23), 10377-10385.

15. Kwon, H.-J.; Seo, J.-A.; Iwahashi, T.; Ouchi, Y.; Kim, D.; Kim, H. K.; Hwang, Y.-H., Study of alkyl chain length dependent characteristics of imidazolium based ionic liquids $[\mathrm{CnMIM}]+[\mathrm{TFSA}]-$ by Brillouin and dielectric loss spectroscopy. Curr. Appl. Phys. 2013, 13 (1), 271-279.

16. Masui, A.; Yoshioka, S.; Kinoshita, S., Light scattering study of liquid and plastic phases of succinonitrile from 0.003 to $3000 \mathrm{~cm}-1$. Chem. Phys. Lett. 2001, 341 (3), 299-305.

17. Das, S.; Bhadram, V. S.; Narayana, C.; Bhattacharyya, A. J., Brillouin Scattering Investigation of Solvation Dynamics in Succinonitrile-Lithium Salt Plastic Crystalline Electrolytes. J. Phys. Chem. B 2011, 115 (43), 12356-12361.

18. Fano, U., Effects of configuration interaction on intensities and phase shifts. Phys. Rev. 1961, $124(6), 1866$. 
19. (a) Henderson, W. A.; Young, V. G.; Passerini, S.; Trulove, P. C.; De Long, H. C., Plastic Phase Transitions in N-Ethyl-N-methylpyrrolidinium Bis(trifluoromethanesulfonyl)imide. Chem. Mater. 2006, 18 (4), 934-938; (b) Forsyth, M.; Huang, J.; MacFarlane, D. R., Lithium doped -methyl-ethylpyrrolidinium bis(trifluoromethanesulfonyl)amide fast-ion conducting plastic crystals. J. Mater. Chem. 2000, 10 (10), 2259-2265.

20. Strange, J. M. C. a. J. H., Diffusion in Molecular Crystals. Physics Reports (Review Section of Physics Letters) 1979, 53(1), 1-92.

21. Jin, H.; O’Hare, B.; Dong, J.; Arzhantsev, S.; Baker, G. A.; Wishart, J. F.; Benesi, A. J.; Maroncelli, M., Physical Properties of Ionic Liquids Consisting of the 1-Butyl-3-methylimidazolium Cation with Various Anions and the Bis(trifluoromethylsulfonyl)imide Anion with Various Cations. $J$. Phys. Chem. B 2011, 115 (5), 1333-1333.

22. Musiał, M.; Malarz, K.; Mrozek-Wilczkiewicz, A.; Musiol, R.; Zorębski, E.; Dzida, M., Pyrrolidinium-Based Ionic Liquids as Sustainable Media in Heat-Transfer Processes. ACS Sustainable Chem. Eng. 2017, 5 (11), 11024-11033.

23. Musiał, M.; Wojnarowska, Z.; Cheng, S.; Ngai, K. L.; Paluch, M., Evidence of a Fundamental Mechanism Governing Conductivity Relaxation in Room-Temperature Ionic Liquid. J. Phys. Chem. C 2019, 123 (36), 22089-22094.

24. Tan, J.-C.; Civalleri, B.; Lin, C.-C.; Valenzano, L.; Galvelis, R.; Chen, P.-F.; Bennett, T. D.; Mellot-Draznieks, C.; Zicovich-Wilson, C. M.; Cheetham, A. K., Exceptionally Low Shear Modulus in a Prototypical Imidazole-Based Metal-Organic Framework. Phys. Rev. Lett. 2012, 108 (9), 095502.

25. Pas, S. J.; Pringle, J. M.; Forsyth, M.; MacFarlane, D. R., Thermal and physical properties of an archetypal organic ionic plastic crystal electrolyte. PCCP 2004, 6 (13), 3721-3725.

26. Fleshman, A. M.; Mauro, N. A., Temperature-dependent structure and transport of ionic liquids with short-and intermediate-chain length pyrrolidinium cations. J. Mol. Liq. 2019, 279, 23-31.

27. (a) Hill, A.; Huang, J.; Efthimiadis, J.; Meakin, P.; Forsyth, M.; MacFarlane, D., Microstructural and molecular level characterisation of plastic crystal phases of pyrrolidinium trifluoromethanesulfonyl 
salts. Solid State Ionics 2002, 154, 119-124; (b) MacFarlane, D. R.; Forsyth, M., Plastic Crystal Electrolyte Materials: New Perspectives on Solid State Ionics. Adv. Mater. 2001, 13 (12-13), 957-966.

28. Pas, S. J.; Huang, J.; Forsyth, M.; MacFarlane, D. R.; Hill, A. J., Defect-assisted conductivity in organic ionic plastic crystals. J. Chem. Phys. 2005, 122 (6), 064704.

29. Brand, R.; Lunkenheimer, P.; Loidl, A., Relaxation dynamics in plastic crystals. J. Chem. Phys. 2002, 116 (23), 10386-10401.

30. Koreeda, A.; Takano, R.; Saikan, S., Light scattering in a phonon gas. Phys. Rev. B 2009, 80 (16), 165104.

31. Henderson, W. A.; Young, V. G.; Pearson, W.; Passerini, S.; Long, H. C. D.; Trulove, P. C., Thermal phase behaviour ofN-alkyl-N-methylpyrrolidinium and piperidinium bis(trifluoromethanesulfonyl)imide salts. J. Phys.: Condens. Matter 2006, 18 (46), 10377-10390.

\section{TABLE}

Table 1. The acoustic velocities of ILs obtained using a refractive index of 1.4 and 1.8 in the liquid phase and phase-I, respectively.

\begin{tabular}{|c|c|c|c|}
\hline \multirow{2}{*}{ Ionic liquid } & \multicolumn{3}{|c|}{ Velocity (m/s) } \\
\cline { 2 - 4 } & Liquid phase & \multicolumn{2}{|c|}{ Phase-I } \\
\cline { 2 - 4 } & LA & LA & TA $_{1}$ \\
\hline P $_{11}$ TFSI & 1064 & 1365 & 447 \\
\hline P $_{12}$ TFSI & 1178 & 1355 & 432 \\
\hline P $_{14}$ TFSI & 1653 & & \\
\hline
\end{tabular}




\section{FIGURES}
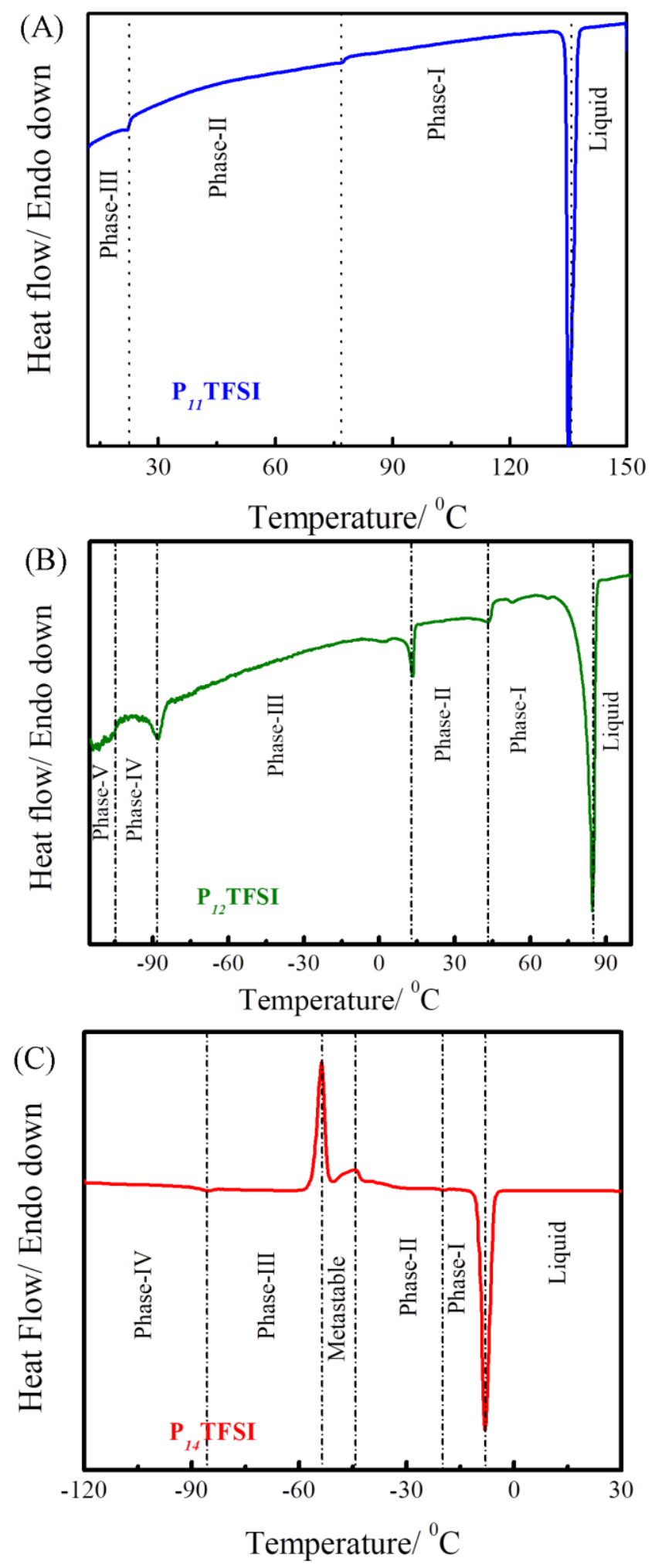

Figure 1. Differential scanning calorimetry (DSC) for (A) $\mathrm{P}_{11}$ TFSI, (B) $\mathrm{P}_{12}$ TFSI, and (C) $\mathrm{P}_{14}$ TFSI at a scan rate of $5{ }^{\circ} \mathrm{C} / \mathrm{min}$. DSC results show melting points and solid-solid transitions. 

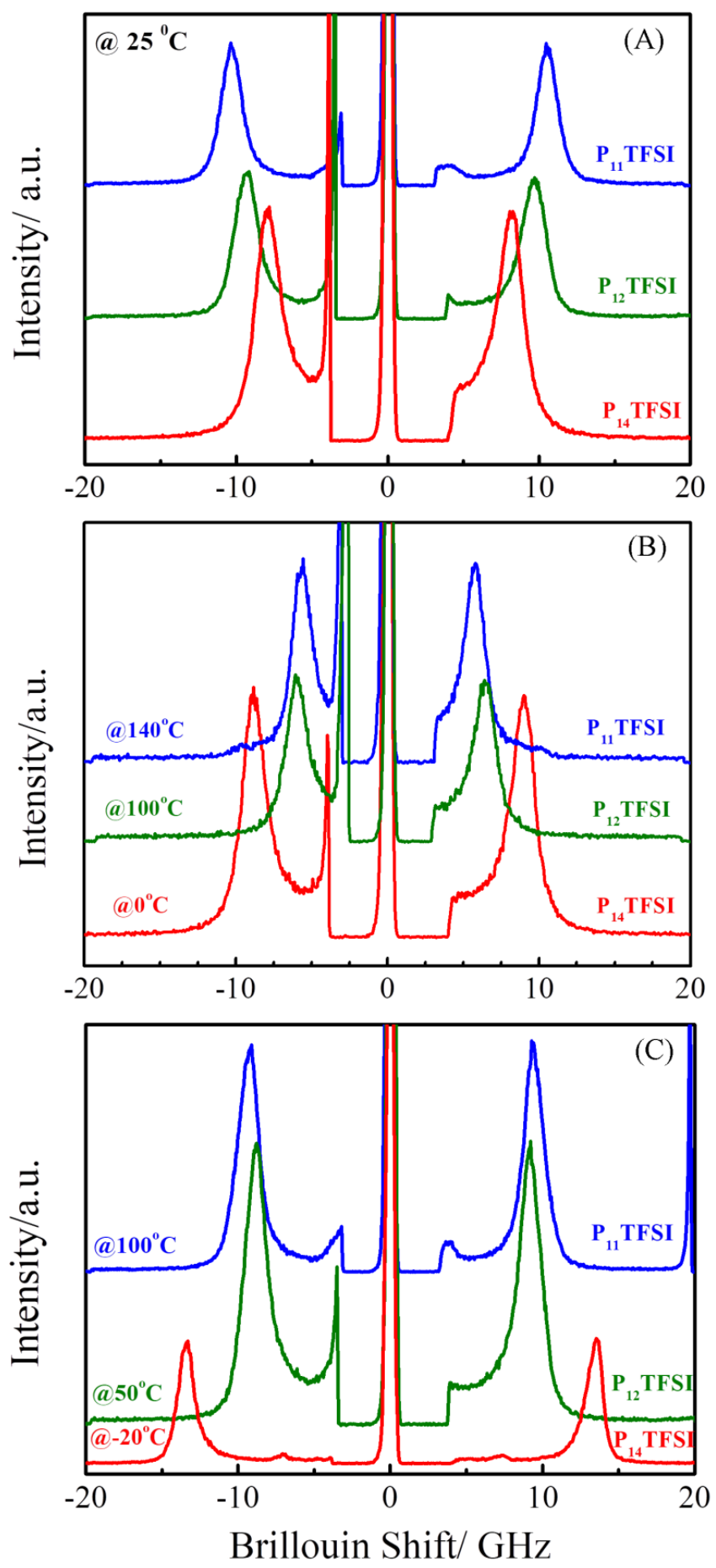

Figure 2. Brillouin spectra of $\mathrm{N}$-alkyl-N-methylpyrrolidinium bis(trifluorosulfonyl) imide $\left(\mathrm{P}_{l n} \mathrm{TFSI}\right.$, $\mathrm{n}=1,2$ and 4$)$ based ionic liquids at different conditions (A) at room temperature $\left(25^{\circ} \mathrm{C}\right)(\mathrm{B})$ in liquid state and (C) in solid-state (phase-I) immediately before melting. 

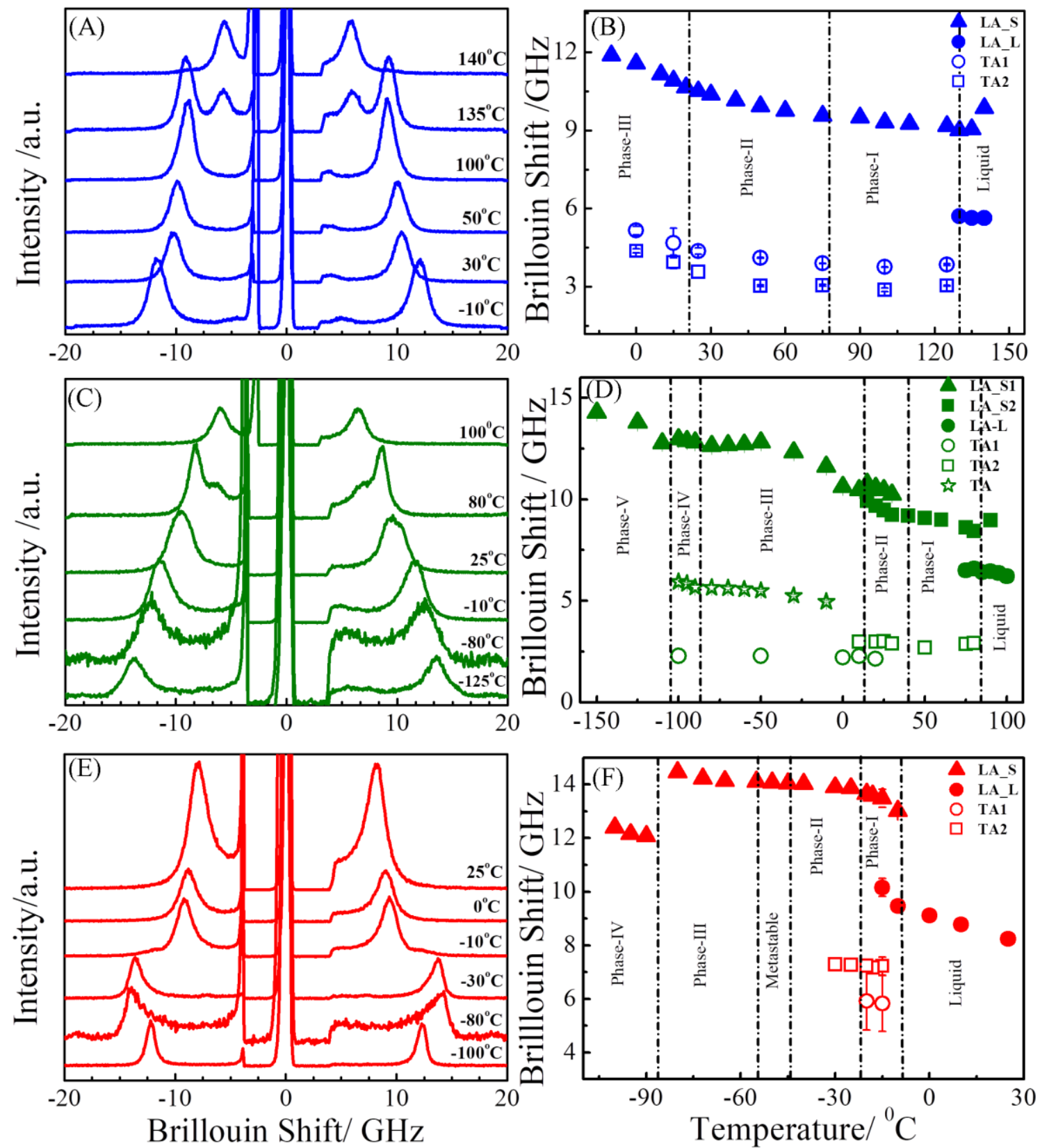

Figure 3. Brillouin spectra of (A) $\mathrm{P}_{11}$ TFSI (C) $\mathrm{P}_{12}$ TFSI and (E) $\mathrm{P}_{14}$ TFSI at various temperatures.

Temperature evolution of Brillouin peak positions (frequencies) of longitudinal and transverse acoustic modes of (B) $\mathrm{P}_{11}$ TFSI (D) $\mathrm{P}_{12}$ TFSI (F) $\mathrm{P}_{12}$ TFSI. LA_S and LA_L correspond to longitudinal acoustic modes in solid and liquid phases, respectively. The vertical dash-dot line indicates the phase transition temperatures according to DSC analysis shown in Figure 1. 


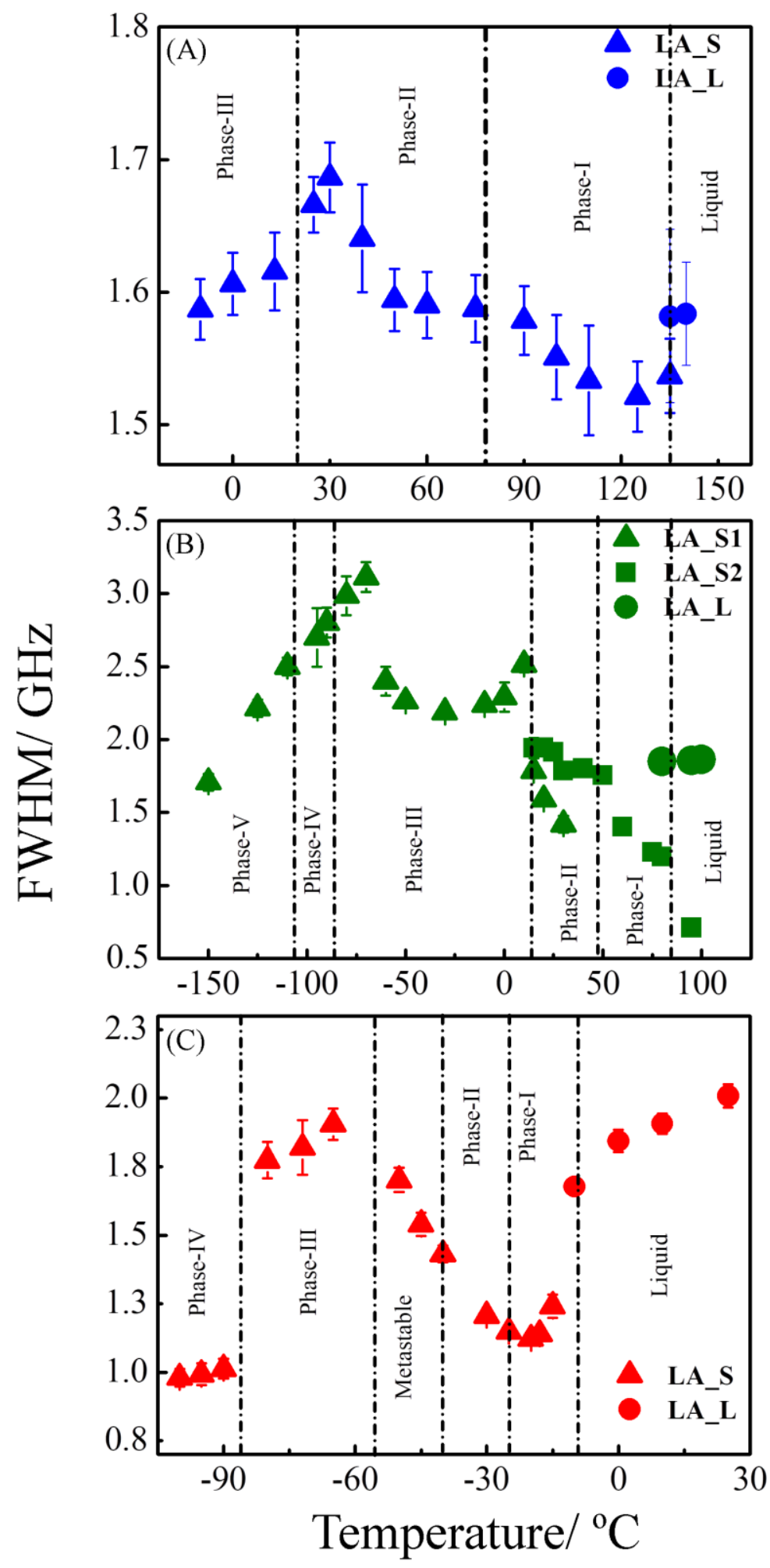

Figure 4. Temperature dependence of the linewidth of (A) $\mathrm{P}_{11}$ TFSI (B) $\mathrm{P}_{12}$ TFSI and (C) $\mathrm{P}_{14}$ TFSI. The vertical short dash-dotted lines designate transition temperature according to DSC data. 

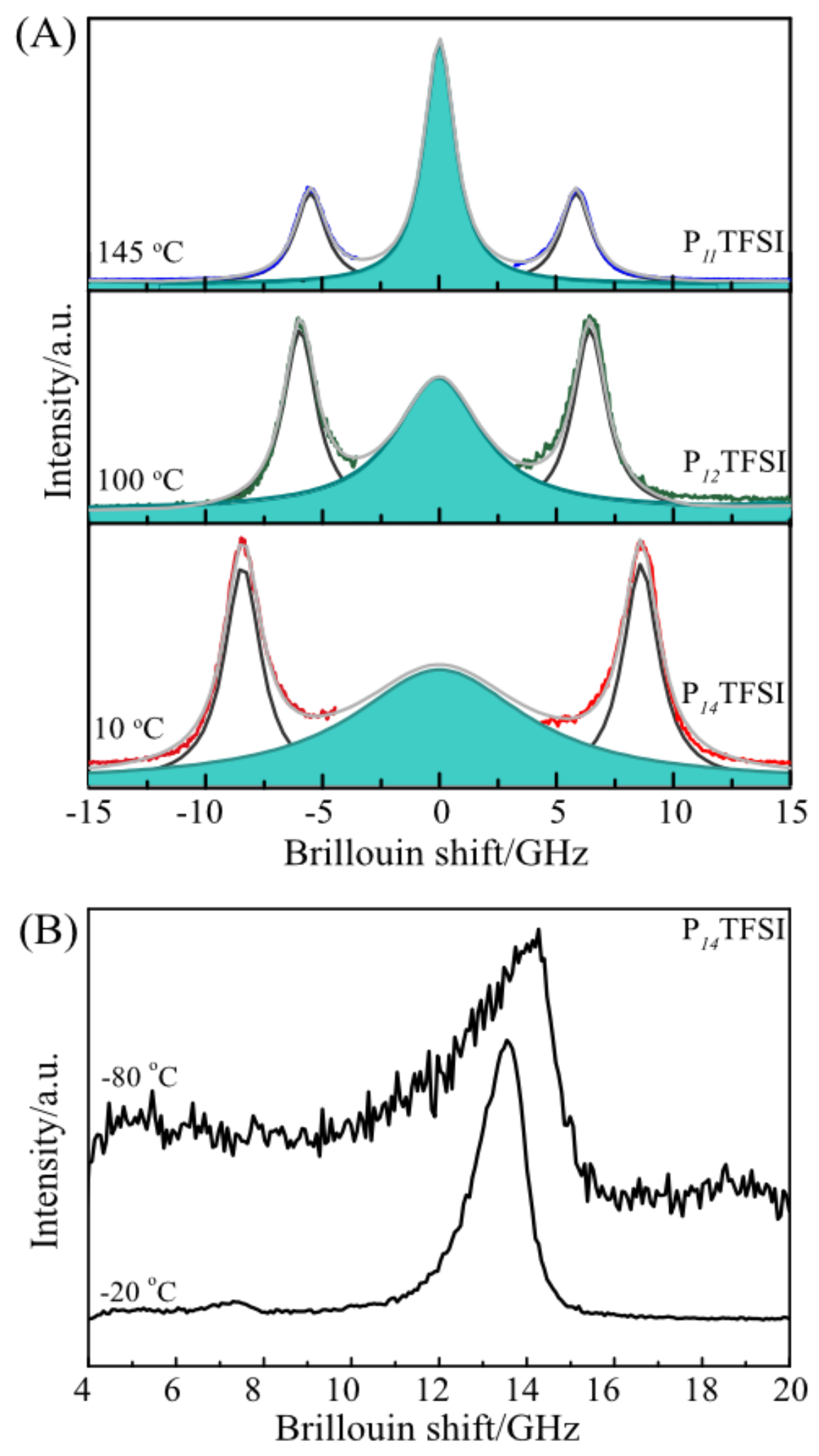

Figure 5. (A) The deconvolution of the central peak (CP) in the liquid phase of ILs (B) Brillouin spectra of $\mathrm{P}_{14}$ TFSI at two different temperatures showing the asymmetric lineshape. 